\section{Diabetic Chorea: A Rare Complication of Poorly Controlled Diabetes Mellitus}

Sir,

A 67-year female was admitted with the complaints of involuntary movements of her left lower limb that had continued for a month. Her medical history revealed she had type-2 diabetes mellitus for 15 years with the complication of ischemic stroke since two years. Her glycemic control was poor. She was taking metformin 2000 $\mathrm{mg} /$ day and insulin glargine 18 ünits/day before; and she had a non-ketotic hyperosmolar coma one month ago, after which her current symptoms emerged. Despite an aggressive treatment of diabetes mellitus, the involuntary movements persisted. On neurological examination, choreiform movements were observed in the left lower limb. The chorea was also persistent during the sleep. She had not taken any medication recently that might cause these movements. Laboratory findings revealed a HbAlc level of $14 \%$. The parameters of complete blood count and urinary tests were in normal ranges. Her cranial magnetic resonance imaging (MRI) did not reveal any intensity changes. However, the computed tomography (CT) revealed a high-density lesion on the right striatum (Figure 1). Based on hermedical history, laboratory, neurological and radiological findings, she was diagnosed as a case of diabetic chorea. The treatment was started with haloperidol $2.5 \mathrm{mg}$ twice a day orally, which was increased to $5 \mathrm{mg}$ after two weeks. Within two weeks of admission, her symptoms regressed remarkably and currently her neurological examination is normal.

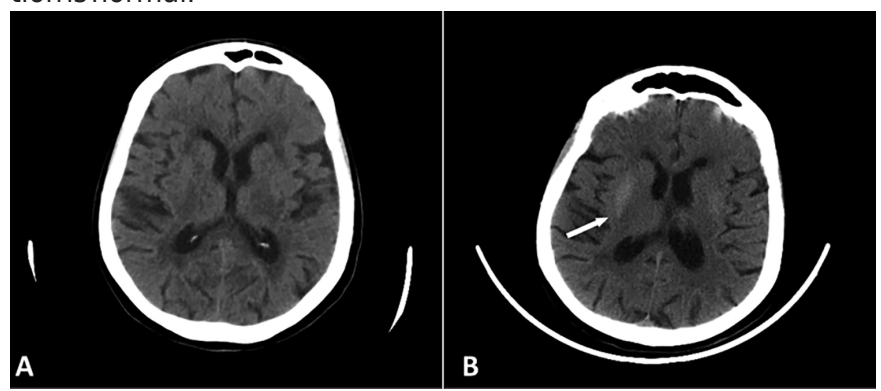

Figure 1: (A) Head CT of the patient taken three months ago, before the diagnosis with non-ketotic hyperosmolar hyperglycemic (NKHH) chorea. (B) After diagnosis of NKHH chorea, a high-density lesion was detected in the rightstriatum (arrow).

In the light of characteristic density changes on neuroimaging, neurological symptoms, and laboratory results, a diagnosis of non-ketotic hyperosmolar hyperglycemic (NKHH) chorea was rendered. NKHH chorea is an infrequent syndrome among patients with diabetes mellitus. ${ }^{1}$ Uncontrolled hyperglycemia may lead to disruption of cerebral autoregulation and trigger dysfunction of the striatum and may manifest as NKHH chorea. ${ }^{1,2}$ The disease presents with involuntary movements and high blood glucose levels accompanied by high density on CT scan and high signal intensity on both $\mathrm{T} 1$ and T2-weighted MRI imaging of the pallidostriatal area. ${ }^{3}$

The exact mechanism of this complication is still uncertain but it is generally believed that cytotoxic edema causes anoxia; therefore, glucose levels diminish in basal ganglia. Other theories implicate the depletion of gamma-aminobutyric acid (GABA) and acetylcholine levels, caused by the absence of acetoacetate or the accumulation of manganese-containing gemistocytes in basal ganglia. ${ }^{3,4}$ The treatment and prevention involve a tight glycemic control; however, when chorea appears, dopamine receptor antagonists can improve the symptoms. It has generally a benign outcome and patients usually have satisfactory prognosis. ${ }^{3}$

This case highlights the need to consider poorly controlled diabetes mellitus in the differential diagnosis of chorea.

\section{CONFLICT OF INTEREST:}

The author declared no conflict of interest.

\section{AUTHOR'S CONTRIBUTION:}

BCA: Conceived, designed, edited and reviewed the final manuscript

\section{REFERENCES}

1. Michael S, Bhowansingh VR. Chorea hyperglycemia basal ganglia syndrome in a 63-year-old male. Case Rep Med 2018; 2018:9101207. doi: 10.1155/2018/9101207.

2. Lin JJ. Ipsilateral putamen hyperintensity on T1-weighted MRI in non-ketotic hyperglycemia with hemiballismhemichorea: A case report. Parkinsonism Relat Disord 2001; 7(4):319-21. doi: 10.1016/s1353-8020(00)00072-9.

3. Xianchao C, Hong W, Yu H, Yao VY. Chorea associated with nonketotic hyperglycemia: A case report with atypical imaging changes. Medicine (Baltimore) 2017; 96(45): e8602. doi: 10.1097/MD.0000000000008602.

4. Eriko M, Shiota H, Watanabe K, Otsuka Y, Yamana M, Yamaguchi $S$, et al. Hemichorea after hypoglycemic episodes with negative mri findings in an elderly woman with poorly controlled type 2 diabetes mellitus: A case report. BMC Neurol 2019; 19(1):131. doi: 10.1186/ s12883-019-1334-2.

Buse Cagla ARI

Siirt State Hospital, Turkey

Correspondence to: Dr. Buse Cagla Ari, Siirt State Hospital, Turkey

E-mail: juvelia@gmail.com

Received: March 02, 2020; Revised: April 19, 2020;

Accepted: May 04, 2020

DOI: https://doi.org/10.29271/jcpsp.2021.02.248 